\title{
REDESCRIPCIÓN DE RHIGONEMA CUBANUM (BARUS, 1969) (RHIGONEMATIDA: RHIGONEMATIDAE) Y DESCRIPCIÓN DE SU ESPERMIOGÉNESIS
}

\author{
Nayla García Rodríguez y Jans Morffe Rodríguez \\ Instituto de Ecología y Sistemática. Carretera de Varona, km 31/2, Capdevila, Boyeros, \\ La Habana, Cuba.nayla@ecologia.cu; jans@ecologia.cu
}

\section{RESUMEN}

Rhigonema cubanum (Barus, 1969) (Nematoda: Rhigonematidae) es redescrita sobre la base de nuevo material procedente de un espirobólido (Diplopoda: Spirobolida) no identificado. Esta especie se caracteriza por presentar un sistema reproductor femenino de Tipo 1, cutícula cervical provista de microtricos visibles hasta el fin del cuerpo esofágico, machos con espículas isomórficas, isométricas, cortas y sigmoidales y ocho pares de papilas: cuatro precloacales y cuatro postcloacales. Se describe la espermiogénesis y se ofrece una tabla comparativa con las medidas de los ejemplares de la localidad tipo y del presente registro.

Palabras clave: Nematoda, Rhigonematida, Rhigonema, espermiogénesis, Diplopoda, Cuba.

\section{ABSTRACT}

Rhigonema cubanum (Barus, 1969) (Nematoda: Rhigonematidae) is redescribed on the basis of new material obtained from an unidentified spirobolid millipede (Diplopoda: Spirobolida). The species is characterized by a Type 1 female genital tract, cervical cuticle bearing a well visible microtricha until the end of corpus, spicules isomorphic, isometric, short and sigmoid and eight pair of papillae: four pre-cloacal and four post-cloacal. The process of spermiogenesis is described. A comparative table with the measurements of the type population and the present record is given.

Key words: Nematoda, Rhigonematida, Rhigonema, spermiogenesis, Diplopoda, Cuba.

\section{INTRODUCCIÓN}

La descripción de Rhigonema cubanum (Barus, 1969) (Nematoda: Rhigonematidae) constituyó el primer registro de un rigonemátido para las Antillas (Barus, 1969). Coy et al. (1993) registraron por segunda vez su presencia en Orthomorpha coarctata (Diplopoda: Polydesmida), hospedero tipo, añadieron a Trigoniulus lumbricinus (Diplopoda: Spirobolida) como nuevo hospedero, y señalaron algunas diferencias de sus especímenes respecto a la descripción original. García et al. (1995) registraron, además, a Rhinocricus duvernoyi (Diplopoda: Spirobolida) como nuevo hospedero. El examen reciente de otros ejemplares asignables a la especie, procedentes de una localidad cercana a la localidad tipo, mostró algunas diferencias morfométricas y morfológicas con los datos originales coincidentes con las variaciones del registro anterior (Coy et al., 1993). Estas diferencias, la relativa brevedad de la descripción y el pequeño y poco detallado formato de las ilustraciones originales determinaron la redescripción de esta especie.

\section{OBJETIVOS}

- Redescribir la especie Rhigonema cubanum (Barus, 1969), un nemátodo del orden Rhigonematida, familia Rhigonematidae.

- Describir la espermiogénesis de esta especie. 


\section{MATERIALES Y MÉTODOS}

Se recolectaron manualmente, bajo hojarasca, varios especímenes de un espirobólido no identificado en la Finca "La Chata", Boyeros, La Habana, Cuba, abril de 2009. Los hospederos se llevaron vivos al laboratorio en frascos con hojarasca del sitio de colecta y se sacrificaron con vapores de éter etílico. Los intestinos se extrajeron inmediatamente mediante un corte en los últimos segmentos del cuerpo. Se realizó su disección en solución salina fisiológica, para extraer los parásitos, que se sacrificaron en agua caliente $\left(60-80^{\circ} \mathrm{C}\right)$ y se fijaron en etanol al $70 \%$.

Los nemátodos se montaron en portaobjetos con glicerina anhidra y se sellaron los bordes del cubreobjetos con esmalte de uñas. La observación se llevó a cabo en un microscopio óptico con aumentos de 40x a 1000x.

Para las mediciones se empleó un micrómetro ocular de escala lineal con una precisión de $0,001 \mathrm{~mm}$, acoplado al microscopio. Las variables morfométricas tomadas fueron las utilizadas por Hunt (2002a). De cada medida se ofrece su rango seguido por la media y la desviación estándar, entre paréntesis, así como el número de estructuras medidas. Todos los valores están expresados en milímetros. Se calcularon los índices de De Man a, b, c y V\%.

Los ejemplares se fotografiaron mediante una cámara digital AxioCam acoplada a un microscopio Carl Zeiss AxiosKop 2. Los dibujos se realizaron en CorelDRAW X3, Corel PHOTO-PAINT X3 y Adobe Photoshop CS2 sobre la base de las microfotografías. Las escalas de las ilustraciones están dadas en milímetros.

El material estudiado se encuentra depositado en la Colección Helmintológica de las Colecciones Zoológicas del Instituto de Ecología y Sistemática, La Habana, Cuba (CZACC) y la Coleçao Helmintologica do Instituto Oswaldo Cruz, Río de Janeiro, Brasil (CHIOC).

\section{RESULTADOS}

\section{TAXONOMÍA}

Familia Rhigonematidae

Género Rhigonema Cobb, 1898

Rhigonema cubanum (Barus, 1969)

Figuras 1, A-H; 2, A-H

Dudekemia cubana Barus, 1969: pp. 269-270, fig. 1, A-D.

Rhigonema cubanum (Barus, 1969) Adamson, 1987: p. 1896.

Diagnosis. Ambos sexos con el anillo cefálico estrecho y poco diferenciado, la cutícula cervical provista de microtricos cortos y finos, visibles desde el final del anillo cefálico hasta el final del cuerpo esofágico. Sistema reproductor femenino de Tipo 1. Cola muy corta y subulada en ambos sexos. Espículas isomórficas y casi isométricas, cortas, curvas y ligeramente sigmoidales. Ocho pares de papilas en posición subventral, cuatro precloacales y cuatro postcloacales.

Diagnosis. Both sexes with cephalic annule strait and poorly differentiated, cervical cuticle bearing short and fine microtricha, well visible from the end of the cephalic annule to the end of corpus. Female genital tract Type 1. Tail very short and subulate in both sexes. Spicules isomorphic, almost isometric, short, curved and slightly sigmoid. Eight pairs of papillae subventral in position, four pre-cloacal and four post-cloacal. 
Medidas. Hembras $(\mathrm{n}=11), \mathrm{a}=10.76-16.64(12.73 \pm 1.64, \mathrm{n}=11) ; \mathrm{b}=8.44-12.37(10.70 \pm 1.50$, $\mathrm{n}=11) ; \mathrm{c}=19.82-37.12(30.16 \pm 5.64, \mathrm{n}=8) ; \mathrm{V} \%=59.46-65.16(62.64 \pm 1.87, \mathrm{n}=6)$; longitud total $=2.700-4.825(3.661 \pm 0.584, \mathrm{n}=11)$; ancho máximo $=0.200-0.360(0.290 \pm 0.048$, $\mathrm{n}=11)$; longitud del cuerpo esofágico $=0.235-0.280(0.254 \pm 0.014, \mathrm{n}=11)$; diámetro del bulbo esofágico $=0.123-0.163(0.142 \pm 0.012, \mathrm{n}=11)$; longitud total del esófago $=0.300-0.390(0.341 \pm$ $0.025, \mathrm{n}=11)$; distancia del anillo nervioso al extremo cefálico $=0.138-0.188(0.161 \pm 0.013, \mathrm{n}=$ $11)$; distancia del poro excretor al extremo cefálico $=0.220-0.260(0.241 \pm 0.013, \mathrm{n}=9)$; distancia de la vulva al extremo de la cola $=1.125-1.800(1.413 \pm 0.225, \mathrm{n}=6)$; longitud del ovoyector $=0.208(\mathrm{n}=1)$; distancia del ano al extremo de la cola $=0.110-0.140(0.123 \pm 0.010, \mathrm{n}=8)$; huevos $=0.080-0.108 \times 0.075-0.090(0.093 \pm 0.006 \times 0.081 \pm 0.004, \mathrm{n}=36)$.

Machos $(n=8), \mathrm{a}=9.92-18.23(13.70 \pm 2.91, \mathrm{n}=8) ; \mathrm{b}=8.87-12.50(9.85 \pm 1.20, \mathrm{n}=8)$; $\mathrm{c}=19.30-28.69(22.77 \pm 3.28, \mathrm{n}=8)$; longitud total $=2.750-4.375(3.197 \pm 0.499, \mathrm{n}=8)$; ancho máximo $=0.170-0.320(0.239 \pm 0.041, \mathrm{n}=8)$; longitud del cuerpo esofágico $=0.233-0.270$ $(0.246 \pm 0.013, \mathrm{n}=8)$; diámetro del bulbo esofágico $=0.118-0.138(0.130 \pm 0.007, \mathrm{n}=8)$; longitud total del esófago $=0.310-0.350(0.324 \pm 0.017, \mathrm{n}=8)$; distancia del anillo nervioso al extremo cefálico $=0.135-0.183(0.161 \pm 0.015, \mathrm{n}=7)$; distancia del poro excretor al extremo cefálico $=0.210-0.260(0.234 \pm 0.021, \mathrm{n}=7)$; distancia del ano al extremo de la cola $=0.120$ $0.153(0.141 \pm 0.010, \mathrm{n}=8)$; longitud de la espícula izquierda $=0.200-0.215(0.209 \pm 0.006, \mathrm{n}=$ $4)$; longitud de la espícula derecha $=0.170-0.228(0.201 \pm 0.017, \mathrm{n}=8)$.

Descripción. Hembras. Nemátodos de mediano tamaño, con el cuerpo robusto. Cutícula con estriaciones transversales muy delgadas. Región cervical provista de microtricos muy cortos y delgados, bien visibles desde el final del anillo cefálico hasta aproximadamente el final del cuerpo esofágico. Abertura bucal trirradial. Labios con cuatro papilas en posición submedia, dos subventrales y dos subdorsales. Anillo cefálico estrecho y poco diferenciado. Esófago típico del género, con el cuerpo esofágico robusto y musculoso, con una expansión distal. Dicha expansión está provista de placas esclerosadas a manera de mandíbulas. Istmo sólo apreciable como constricción entre el cuerpo y el bulbo esofágico, también musculoso y casi esférico, algo achatado y anclado a la pared del cuerpo por haces de fibras musculares que se unen a su parte más ancha. Tres cardia se proyectan desde el bulbo hacia el intestino. Una hilera de células redondeadas y parduscas rodea el cuerpo esofágico tras la dilatación distal. Intestino subrectilíneo y simple. Anillo nervioso aproximadamente en la región media del cuerpo esofágico. Poro excretor situado ligeramente anterior a la unión del cuerpo esofágico con el bulbo esofágico. Vulva no sobresaliente, algo desplazada hacia la mitad posterior del cuerpo. Vagina corta, muy musculosa, sin divertículo, dirigida anteriormente. Sistema reproductor didelfo-anfidelfo, Tipo 1, según Adamson (1987). Ovarios reflexos. Huevos numerosos, elipsoides, grandes y de cáscara lisa. Cola subulada, corta y recta.

Machos. Menores que las hembras. Región cefálica y sistema digestivo similar a las hembras. Espículas isomórficas, casi isométricas, cortas, muy ligeramente sigmoidales, curvadas ventralmente. Región distal de las espículas terminada en punta, cubierta por un velo cuticular. Superficie lateral de las espículas provista de una depresión longitudinal ornamentada por punteaduras finas. Músculos protractores de las espículas muy desarrollados, dispuestos en un haz grueso anclado a la región ventral de la cola. Papilas caudales: cuatro pares precloacales más o menos equidistantes entre sí, en posición subventral, uno de ellos muy próximo al labio anterior de la cloaca y cuatro pares postcloacales, también subventrales. Cola muy corta, subulada y curvada ventralmente. Testis reflexo a una distancia de cerca de tres veces el ancho del cuerpo tras el esófago. Espermiogénesis similar a la descrita por Adamson (1987) en machos de R. seychellarum Adamson, 1987. 

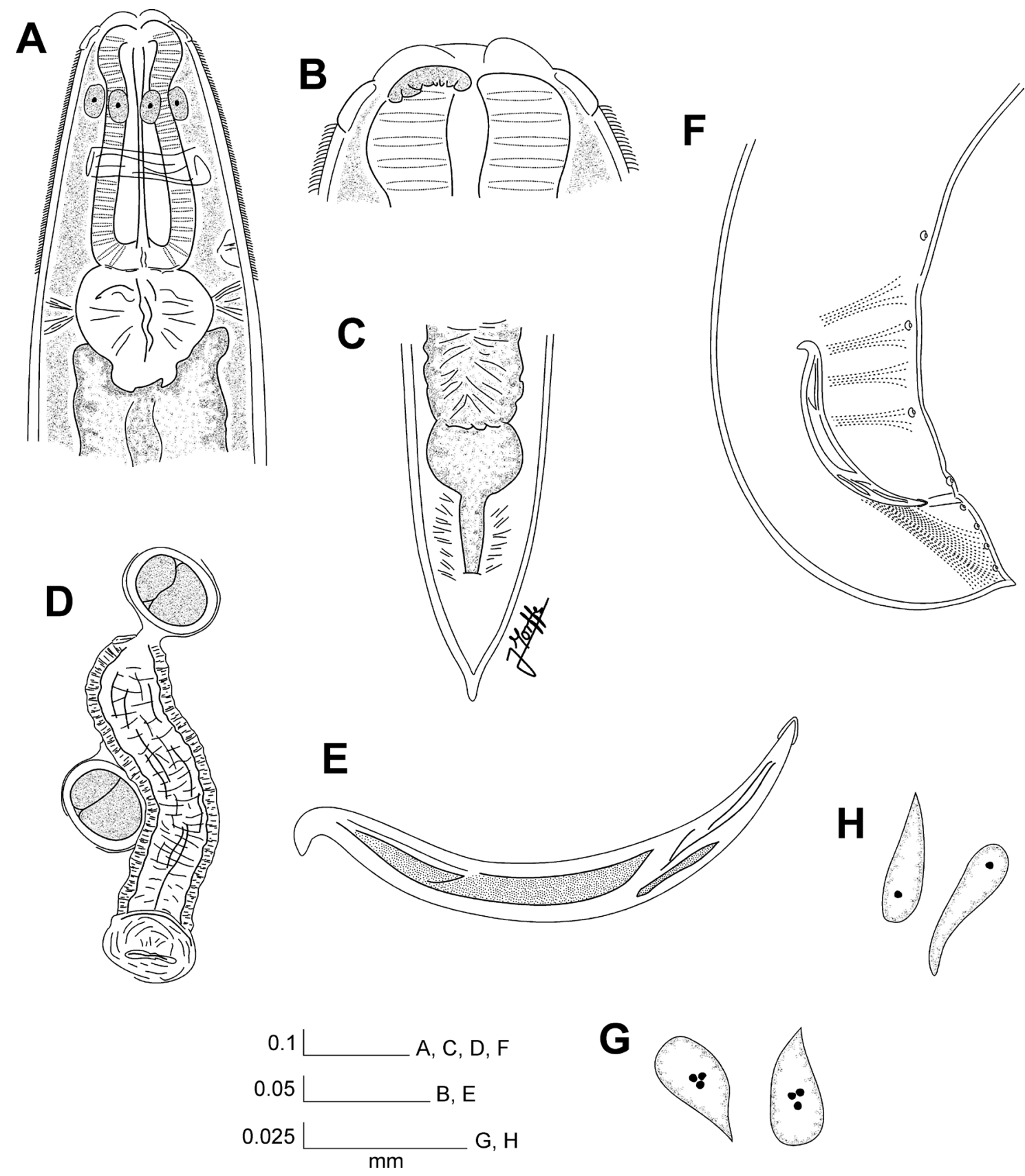

Figura 1. Rhigonema cubanum (Barus, 1969). A. Región esofágica de la hembra, vista lateral. B. Extremo cefálico de la hembra. C. Cola de la hembra, vista dorso-ventral. D. Ovoyector y huevos, vista ventral. E. Espícula derecha, vista lateral. F. Cola del macho, vista lateral. G, H. Espermátidas. 

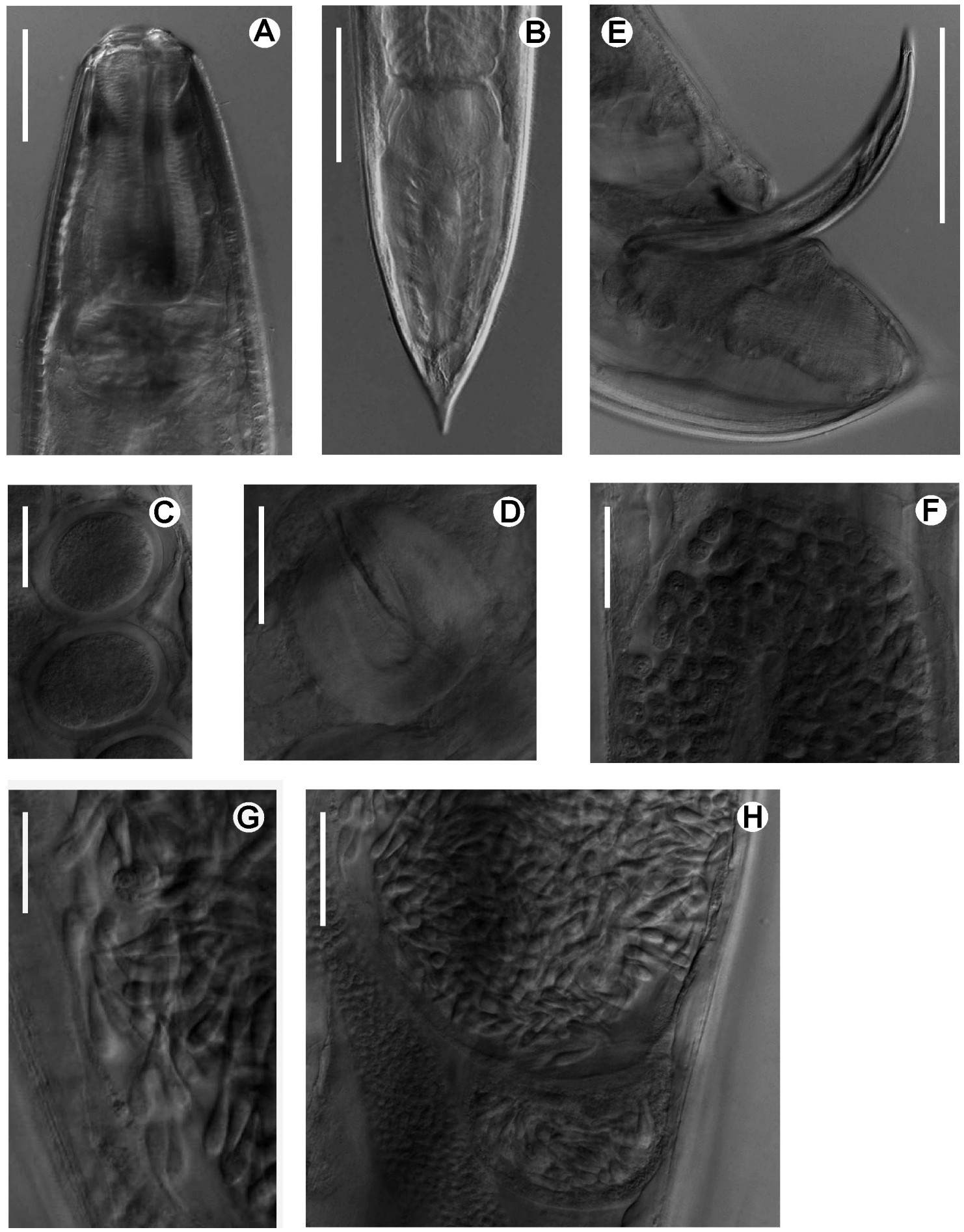

Figura 2. Rhigonema cubanum (Barus, 1969) microfotografías. A. Región esofágica de la hembra, vista lateral. B. Cola de la hembra, vista dorso-ventral. C. Huevos. D. Vagina. E. Espícula derecha parcialmente evertida. F. Espermatocitos al nivel de la flexión del testis. G. Espermátidas. H. Espermátidas acumuladas hacia el final del testis. Líneas de escala: A, B, 0.1 mm. C, D, E, F, H, 0.05 mm. G, $0.025 \mathrm{~mm}$ 
Espermatocitos primarios en el extremo posterior del testis, se aprecian como células redondeadas, de apariencia granulosa, con núcleos prominentes, en los que se distinguen grandes nucléolos redondeados. A lo largo del testis y hacia la región flexionada, los espermatocitos se agrandan y el núcleo se compacta. Ambas estructuras corresponden a la primera división meiótica. A continuación se observan espermátidas con diferentes grados de desarrollo, con forma de gotas de agua, muy alargadas, no es posible distinguir el material nuclear. No se observan las células multilobuladas, referidas por Adamson (1987).

Material examinado. Nueve (9) hembras; CZACC 11.4631-11.4639; en espirobólido no identificado (Diplopoda: Spirobolida); Finca "La Chata", Boyeros, La Habana, Cuba; abril de 2009; J. Morffe col. Seis (6) machos; CZACC 11.4640-11.4645; idénticos datos que las hembras. Dos (2) hembras y dos (2) machos; CHIOC; idénticos datos que los anteriores.

Hospedero. Espirobólido no identificado (Diplopoda: Spirobolida).

Localización. Intestino posterior.

Localidad del presente registro. Finca "La Chata", Boyeros, La Habana, Cuba.

Distribución. Arroyo Naranjo, La Habana, Cuba; Finca “La Chata”, Boyeros, La Habana, Cuba; El Salón, Artemisa, Cuba.

\section{DISCUSIÓN}

Rhigonema cubanum pertenece al grupo de especies con sistema reproductor del Tipo 1, según la clasificación establecida por Adamson (1987), caracterizado por la carencia de divertículo y la presencia de una vagina corta y musculosa. Se diferencia de $R$. longeacauda (Travassos et Kloss, 1959), $R$. falcatum (Artigas, 1926), $R$. chanaae (Travassos et Kloss, 1960), R. perezassoi García et Fontenla, 2002 y R. paraiso García et Fontenla, 2004 (Artigas, 1926; García y Fontenla, 2002, 2004; Travassos y Kloss, 1959, 1960) por la presencia de microtricos. La forma de las espículas, cortas y ligeramente sigmoidales, la diferencia de R. multipapillatum (Skrjabin, 1916), R. rhuti (Travassos et Kloss, 1960), $R$. disparovis Van Waerebeke, 1991, R. piedralavela García, Coy et Ventosa, 1998, y $R$. peziphorum Hunt, 2002, que poseen espículas alargadas, estrechas y curvas las cuatro primeras y cortas, pero no sigmoidales en la última (García et al., 1998; Hunt, 2002a, b; Travassos y Kloss, 1960; Van Waerebeke, 1991). De R. prolifica (Bowie, 1985), R. alpinensis (Bowie, 1985), R. kaorinus (Bowie, 1985) y R. hirsutus (Bowie, 1985), se distingue por la mayor abundancia y extensión de los microtricos, hasta dos tercios de la longitud total del cuerpo en las especies neozelandesas (Bowie, 1985).

De R. glabrum (Dollfus, 1952), R. longispicula (Travassos et Kloss, 1959), R. sooretama (Travassos et Kloss, 1960), R. aguirrei (Travassos et Kloss, 1960), R. acuminatum (d'Udekem, 1859), R. ornatum (Majumdar, 1967) y R. travassosi (Dollfus, 1964), especies no ubicadas por Adamson (1987) en el grupo con aparato genital Tipo 1 pero carentes de divertículo vaginal (Hunt, 1996), difiere por la ausencia de microtricos en las cuatro primeras y por la forma y longitud de las espículas en las tres últimas (Dollfus 1952, 1964; d’Udekem, 1859; Travassos y Kloss, 1959, 1960; Barus, 1969; Van Waerebeke, 1984). R. leonilae (Sánchez-Velázquez, 1979) y $R$. caballerodeloyai (Sánchez-Velázquez, 1979), que también pudieran ser consideradas dentro del Tipo 1 según las características de su aparato reproductor, se diferencian por la forma y longitud de las espículas.

Las medidas de los ejemplares de la población estudiada no difieren mucho de las registradas por Barus (1969) para la localidad tipo (Tabla 1). Sólo la distancia del anillo nervioso al extremo 
Tabla 1. Medidas de las hembras y machos de Rhigonema cubanum (Barus, 1969) de Arroyo Naranjo, La Habana, Cuba (localidad tipo) y la Finca La Chata, Boyeros, La Habana, Cuba.

\begin{tabular}{|c|c|c|}
\hline Hospedero & $\begin{array}{l}\text { Ortomorpha coarctata } \\
\text { (Diplopoda: Polydesmida) }\end{array}$ & $\begin{array}{l}\text { Espirobólido no identificado } \\
\text { (Diplopoda: Spirobolida) }\end{array}$ \\
\hline Localidad & $\begin{array}{l}\text { Arroyo Naranjo, La Habana (localidad } \\
\text { tipo) }\end{array}$ & $\begin{array}{l}\text { Finca "La Chata", Boyeros, } \\
\text { La Habana }\end{array}$ \\
\hline Medidas de las hembras & $\mathrm{n}=2$ & $\mathrm{n}=11$ \\
\hline Longitud total & $3.930-4.080$ & $2.700-4.825$ \\
\hline Ancho máximo & $0.320-0.420$ & $0.200-0.360$ \\
\hline Longitud del cuerpo esofágico & $0.260^{*}$ & $0.235-0.280$ \\
\hline Diámetro del bulbo esofágico & No se ofrecen medidas & $0.123-0.163$ \\
\hline Longitud del esófago & $0.340-0.420$ & $0.300-0.390$ \\
\hline Anillo nervioso-extremo anterior & $0.087^{*}$ & $0.138-0.188$ \\
\hline Poro excretor-extremo anterior & 0.260 & $0.220-0.260$ \\
\hline Vulva-cola & $1.460-1.530$ & $1.125-1.800$ \\
\hline Ano-cola & 0.120 & $0.110-0.140$ \\
\hline Huevos & $0.094-0.102 \times 0.073-0.087$ & $0.080-0.108 \times 0.075-0.090$ \\
\hline a & $9.71-12.28$ & $10.76-16.64$ \\
\hline $\mathrm{b}$ & $9.71-11.56$ & $8.44-12.37$ \\
\hline $\mathrm{c}$ & $32.75-34.00$ & $19.82-37.12$ \\
\hline $\mathrm{V} \%$ & $62.50-62.85$ & $59.46-65.16$ \\
\hline Medidas de los machos & $\mathrm{n}=9$ & $\mathrm{n}=8$ \\
\hline Longitud total & $1.970-3.930$ & $2.750-4.375$ \\
\hline Ancho máximo & $0.250-0.320$ & $0.170-0.320$ \\
\hline Longitud del cuerpo esofágico & 0.220 & $0.233-0.270$ \\
\hline Diámetro del bulbo esofágico & 0.110 & $0.118-0.138$ \\
\hline Longitud del esófago & $0.250-0.330$ & $0.310-0.350$ \\
\hline Anillo nervioso-extremo anterior & 0.080 & $0.135-0.183$ \\
\hline Poro excretor-extremo anterior & 0.240 & $0.210-0.260$ \\
\hline Ano-cloaca & 0.120 & $0.120-0.153$ \\
\hline Espícula izquierda & $0.175-0.211 * *$ & $0.200-0.215$ \\
\hline Espícula derecha & & $0.170-0.228$ \\
\hline a & $7.88-12.28$ & $9.92-18.23$ \\
\hline $\mathrm{b}$ & $7.88-11.91$ & $8.87-12.50$ \\
\hline $\mathrm{c}$ & $16.42-32.75$ & $19.30-28.69$ \\
\hline
\end{tabular}

*Solo se ofrece la medida del holotipo.

**Se ofrecen las medidas de las espículas en general, sin especificar el lado al que pertenecen. 
anterior es mayor en nuestros ejemplares que la registrada por Barus (0.138-0.188 vs. 0.087 en las hembras y $0.135-0.183$ vs. 0.080 en los machos). Las medidas de dicho autor parecen coincidir con la hilera de células glandulares que rodea el cuerpo esofágico, situada por delante del propio anillo nervioso.

\section{AGRADECIMIENTOS}

Agradecemos al MSc. Yamir Arias (Instituto de Ecología y Sistemática) por su ayuda en la obtención de las microfotografías. Al Dr. Luis F. de Armas (Instituto de Ecología y Sistemática) por la revisión del manuscrito. Al MSc. José M. Guzmán, por su apoyo logístico. Este trabajo está apoyado por IDEAWILD y el proyecto "Colecciones zoológicas, su conservación y manejo" del Ministerio de Ciencia, Tecnología y Medio Ambiente (CITMA), Cuba.

\section{LITERATURA CITADA}

Adamson, M. A. 1987. Rhigonematid (Rhigonematida; Nematoda) parasites of Scaphiostreptus seychellarum (Spirostreptida: Diplopoda) in the Seychelles with comments on oveyector structure in Rhigonema Cobb, 1898. Can. J. Zool. 65: 1889-1897.

Artigas, P. 1926. Nematodes de invertebrados (IV). Bol. Biol. Sao Paulo 1 (4): 97-110.

Barus, V. 1969. Dudekemia cubana sp. n. (Nematoda; Rhigonematidae), from the Cuban millipede Orthomorpha coarctata. Folia Parasitol. 16: 269-270.

Bowie, J. Y. 1985. New species of rhigonematid and thelastomatid nematodes from indigenous New Zealand millipedes. New Zealand J. Zool. 12: 485-503.

Coy, A., N. García y M. Álvarez. 1993. Nemátodos parásitos de diplópodos cubanos con descripción de nueve especies, siete de ellas nuevas. Acta Biol. Venez. 14: 33-51.

Dollfus, R. Ph. 1952. Quelques Oxyuroidea de Myriapodes. Ann. Parasitol. Hum. Comp. 27: 143-326.

Dollfus, R. Ph. 1964. Nematodes de Myriapodes du Congo Belge. Mem. Mus. Nat. Hist. Natur. Paris 32: 109-169.

d'Udekem, M. 1859. Notice sur quelques parasites du Julius terrestris. Bull. Ac. Royal. Sci. Belgique 2 (7): 552-567.

García, N., A. Coy y M. Álvarez. 1995. Nuevo género y nuevas especies de nemátodos (Nematoda) parásitos de artrópodos cubanos. Poeyana 449: 1-14.

García, N., A. Coy y L. Ventosa. 1998. Primer registro de nemátodos (Nematoda; Oxyurida; Rhigonematida) parásitos de Amphelictogon sp. (Diplopoda; Polydesmida) para Cuba. Avicennia 8/9: 50-56.

García, N. y J. L. Fontenla. 2002. New species of nematodes (Nematoda: Rhigonematida) associated with Rhinocricus parcus (Diplopoda). Solenodon 2: 1-5.

García, N. y J. L. Fontenla. 2004. Especies nuevas de nemátodos (Nematoda: Rhigonematida) asociados con Anadenobolus a. arboreus (Diplopoda: Rhinocricidae) de Puerto Rico. Solenodon 4: 6-10. 
Hunt, D. J. 1996. A synopsis of the Rhigonematidae (Nematoda), with an outline classification of the Rhigonematida. Afro-Asian J. Nematol. 6 (2): 137-150.

Hunt, D. J. 2002a. The African Rhigonematoidea (Nematoda: Rhigonematida). 2. Six new species of Rhigonema Cobb, 1898 (Rhigonematidae). Nematology 4 (7): 803-827.

Hunt, D. J. 2002b. The African Rhigonematoidea (Nematoda: Rhigonematida).1. Trachyglossus quintus gen. n., sp. n. (Xustrostomatidae fam. n.) with redescription of Rhigonema multipapillatum (Skrjabin, 1916) and designation of a neotype. Nematology 4 (6): 671-686.

Sánchez-Velázquez, L. 1979. Seis nuevas especies de nemátodos parásitos de diplópodos de México. An. Inst. Biol. Univ. Nal. Autón. México 50 (1): 63-117.

Travassos, L. y G. Kloss. 1959. Sobre a familia Rhigonematidae (Artigas, 1930). Atas Soc. Biol. Rio de Janeiro. 3 (5): 9-10.

Travassos, L. y G. Kloss. 1960. Alguns nematóides de familia Rhigonematidae (Artigas, 1930). Atas Soc. Biol. Rio de Janeiro. 4 (1): 2-5.

Van Waerebeke, D. 1984. Rhigonema madecassum n. sp. (Rhigonematidae; Nematoda), parasite de Diplopode à Madagascar: description et étude de la spermiogenèse. Revue Nematol. 7 (3): 271-276.

Van Waerebeke, D. 1991. Rhigonema disparovis n. sp. (Rhigonematidae, Nematoda) parasite de Pachybolus laminatus (Diplopoda) en Cote D'Ivoire. Revue Nematol. 14: 95-100. 